\title{
Strain rate sensitivity of sintered silver nanoparticles using rate-jump indentation
}

\author{
Xu Long ${ }^{\mathrm{a}, *}$, Wenbin Tang ${ }^{\mathrm{a}}$, Yihui Feng ${ }^{\mathrm{b}}$, Chao Chang ${ }^{\mathrm{c}}$, Leon M. Keer ${ }^{\mathrm{d}}$, Yao Yao ${ }^{\mathrm{a}, *}$ \\ a School of Mechanics, Civil Engineering and Architecture, Northwestern Polytechnical University, Xi'an 710072, China \\ ${ }^{\mathrm{b}}$ State Key Laboratory of Nonlinear Mechanics, Institute of Mechanics, Chinese Academy of Sciences, Beijing 100190, China \\ c School of Applied Science, Taiyuan University of Science and Technology, Taiyuan, China \\ ${ }^{\mathrm{d}}$ Department of Civil and Environmental Engineering, Northwestern University, 2145 Sheridan Rd., Evanston, IL 60208, USA
}

\section{A R T I C L E I N F O}

\section{Keywords:}

Silver nanoparticles

Strain rate sensitivity

Nanoindentation

Strain rate sensitivity

Creep strain rate

Stress exponent

\begin{abstract}
A B S T R A C T
Nanoindentation experiments were performed at room temperature on pressure-less sintered silver nanoparticles (AgNP) samples. Two representative die attach solder materials: sintered electrically conductive silver adhesive and conventional Sn-3.0Ag-0.5Cu solder were investigated. A novel technique of multiple strain-rate jumps is adopted accompanied by the continuous stiffness measurement, which can effectively determine the strain rate sensitivity (SRS) with good accuracy. Different strain rates and indentation depths are considered to obtain the nanomechanical properties such as hardness and Young's modulus. Compared with Young's modulus of sintered AgNP, the effect of applied strain rate is more influential to the hardness. In the loading stage, the SRS exponent decreases due to a smaller variation of hardness at a greater indentation depth. In the holding stage prior to the unloading of the applied indentation force, the creep displacement is relatively insensitive to the applied strain rate, however, the creep strain rate decays exponentially and the corresponding stress exponents are determined.

(C) 2018 Elsevier Ltd. All rights reserved.
\end{abstract}

\section{Introduction}

In electrical and electronic equipment, lead-free solders are replacing conventional lead-rich solder alloys as legislated by RoHS [1] due to the concerns about environment and human health. The $\mathrm{Sn}-\mathrm{Ag}-\mathrm{Cu}$ series lead-free solder materials have been popular in applications of the last decade due to outstanding mechanical and electrical properties [2]. Compared with tin-based solder materials, sintered silver paste with nanoparticles own superior electrical and thermal conductivities and therefore have been considered as one of the promising lead-free die attach materials for high-power electric devices where the working temperature can be greater than $250^{\circ} \mathrm{C}[3,4]$. Because of the high thermal conductivity of silver and low temperature sintering properties of silver nanoparticles (AgNP), ultra-low thermal resistance of thermal interface materials can be achieved [5]. With organic shells of citrates covering the nanoparticles to stabilize AgNP, Cu-to-Cu interconnects are feasible based on the pressure-less low temperature sintering of AgNP for electronic packaging [6]. More importantly, the reliability issues about sintered AgNP have been attracting intensive attention recently.

Regarding the performance of pressure-less AgNP joint during longterm reliability, Chua and Siow [7] characterized the evolution of porosity and microstructure of the pressure-less sintered AgNP joints

\footnotetext{
* Corresponding authors.

E-mail addresses: xulong@nwpu.edu.cn (X. Long), yaoy@nwpu.edu.cn (Y. Yao).

on $\mathrm{Cu}$, direct bond copper and $\mathrm{Ag}$ plated substrates during their high-temperature storage in air at $300{ }^{\circ} \mathrm{C}$. Regarding the morphology changes, Paknejad et al. [8] found that the exterior sintered silver surface oxidizes preventing surface morphology changes, and the interior pore surfaces of the porous silver can remain largely oxide-free, which facilitates fast atomic movement resulting in grain growth and changes in the internal microstructure. If a passivating layer on the interior pores forms, the sintered AgNP is speculated to be suitable to those urgent applications up to $400{ }^{\circ} \mathrm{C}$. By doping tin (up to 10 w.t.\%) to silver paste, Yang et al. [9] chose $235{ }^{\circ} \mathrm{C}$ as the highest sintering processing temperature based on the transient liquid phase sintering. A pressure-less bonding strength of $23 \mathrm{MPa}$ is achieved due to the second-phase strengthening mechanism achieved by forming the composite microstructure of silver matrix grains, which are reinforced by $\mathrm{Ag}_{3} \mathrm{Sn}$ and solid solution strengthening mechanism. This is done by forming the Ag-Sn solid solution within the matrix grains. Compared with as-sintered silver bulk, Gadaud et al. [10] studied the quantitative evolution of pores and grains of sintered AgNP and their effects on Young's modulus, yield strength and ultimate tensile stress under the isothermal conditions at $125^{\circ} \mathrm{C}$ up to $1500 \mathrm{~h}$ or under thermal cycling. Based on analytical and computational FEA approaches, Leslie et al. [11] estimated the viscoplastic properties for heterogeneous morphologies of an adhesive-based particulate composite for low temperature applications and a porous sintered version for high-temperature applications. Nevertheless, the strain rate sensitivity of pressure-less sintered AgNP based on the mechanical properties and creep behavior using indentation are not well documented to 
the best of the authors' knowledge and the related experimental results to date are scarce.

\section{Material and method}

In the current study, silver sintering paste is from Fairfield USA Inc., which has a pressure-less sintering capability and outstanding interface reliability due to its resin dispersion system. The sintered AgNP samples are obtained under the curing condition provided by a sintering furnace at the temperature of $200{ }^{\circ} \mathrm{C}$ with 90 min dwelling time and a ramp rate of $2.5^{\circ} \mathrm{C} / \mathrm{min}$. In order to compare the property difference due to microstructure morphology, the control sample made of electrically conductive silver adhesive (ECA) is cured similarly. The 96.5Sn-3.0Ag$0.5 \mathrm{Cu}$ (in w.t.\%, SAC305) bulk solder alloy was obtained from Alpha Assembly Solutions. The solder bars are manufactured using high purity raw materials to avoid impurities, and by using viscosity and dross lowering treatment, the alloy is conditioned to be free of cast in impurities or included oxides.

The material samples for nanoindentation tests were thermally annealed at $210{ }^{\circ} \mathrm{C}$ for $12 \mathrm{~h}$ in an air furnace with a temperature stability of $\pm 1.0^{\circ} \mathrm{C}$ by referring to the optimized condition to remove residue stress of SAC305 bulk solder alloy [12]. After thermal annealing treatment, the samples were mounted in PVC tubes by dental base acrylic resin powder. With a favorably convenient process, the dental base acrylic resin powder can solidify at room temperature without releasing much heat, so that the influence of temperature to the samples is avoided. In addition, the stiffness of solidified acrylic resin is satisfactory, which provides a matrix for material samples during indentation tests. Subsequently, the prepared samples were mechanically ground by $\mathrm{SiC}$ abrasive paper, polished with a metal diamond suspension of $0.5 \mu \mathrm{m}$, and eventually ultrasonically cleaned in ethanol. After being air dried, the obtained samples were stored at room temperature for 7 days to achieve a stable microstructure before indentation tests.

Using the laser flash method, the thermal conductivity of the AgNP sample was measured to be up to $223.91 \mathrm{~W} / \mathrm{m} \cdot \mathrm{K}$, which is much higher than ECA and SAC305 solder as reported by other researchers [13]. Fig. 1 shows the microstructural morphology of material samples in this study. As the SAC305 solder sample is a mirror-like surface after carefully polishing, the SAC305 solder has a highly smooth morphology in Fig. 1(c). Although the same polishing procedure was performed for all samples, the surface of sintered AgNP and ECA has randomly distributed voids. This is due to the entrapped outgassing, which was produced when the solvent evaporates and rheological additives occurred in the solder paste during heating processes [14]. Compared with sintered ECA in Fig. 1(b), the clustered silver particles are sintered with fewer internal voids as shown in Fig. 1(c). Since both AgNP and ECA are mainly composed of silver, the distinguishing difference is induced by the silver morphology and the superior thermal conductivity of sintered AgNP stems from the condensed microstructure with a lower porosity.

In addition to the higher porosity, the sintered ECA with silver flakes does not take full advantage of material or paths for thermal and electrical conductions, compared with sintered AgNP. According to the schematic sintering mechanisms in Fig. 2, the nanoparticles will greatly expand the area of intermetallic bonds between silver particles and therefore benefit the electrical and thermal conductions. Thus, abundant conduction paths in sintered AgNP lead to much higher electrical and thermal conductivities. This is one of the most outstanding advantages of sintered AgNP as a popular die attach bonding material of the electronic packaging structures especially for high-temperature power devices.

Using the Nano Indenter G200 by Agilent Technologies, a three-sided Berkovich diamond indenter was adopted for an identical pattern of ten points on each sample. The indentation points were sufficiently spaced to avoid the interaction of deformation and ensure a high degree of accuracy and precision. The dependence of nanomechanical properties (such as hardness and Young' modulus) on strain rates are of interest, however, a low strain rate applied from the beginning of indentation means time cost for each indentation is unaffordable. Therefore, a strain-rate jump technique proposed by Maier et al. [15] was adopted to examine the strain-rate sensitivity (SRS) of sintered AgNP materials. Compared with the indentation with a constant strain rate, the technique with strain-rate jumps can initiate the indentation process with a relatively high strain rate and subsequently jump to a desired rate level at a preferred indentation depth for multiple times. The rate-jump technique greatly saves the indentation time and avoids the inaccuracy due to thermal drift correction [16], especially for very low indentation depths influenced by surface roughness. More importantly, the SRS effect can be effectively investigated for multiple stain rates at an identical indentation location and therefore the inhomogeneity of material distribution throughout the samples can be ruled out. In fact, Alkorta et al. [17] compared conventional nanoindentation creep experiments with nanoindentation strain-rate jump experiments and found that the resolution and accuracy of the strain-rate jump nanoindentation experiments are unaffected by thermal drift rate and indentation depth and is highly preferable to investigate the nanomechanical properties of soft materials such as solder materials. In this study, the initial strain rates of $0.1 \mathrm{~s}^{-1}$ and $0.2 \mathrm{~s}^{-1}$ were applied for each material sample at room temperature and the applied values were also measured on the indenter. After $1100 \mathrm{~nm}$, three strain-rate jumps were assigned and each strain rate lasted for $150 \mathrm{~nm}$ in the controlled strain rate history until the maximum indentation depth of $2000 \mathrm{~nm}$. Later, a holding stage of $20 \mathrm{~s}$ was applied to investigate the creep effect prior to the unloading stage. For objectiveness, the same procedure was followed for the nanoindentations on all samples.

When measuring indentation response, the Poisson's ratio for each kind of material sample has to be assigned as an input parameter. Regarding the SAC305 solder sample, the Poisson's ratio of 0.42 is adopted as reported by Nguyen et al. [18], where the temperature dependence of Poisson's ratio was found to be negligible over the temperature range between $25^{\circ} \mathrm{C}$ and $105^{\circ} \mathrm{C}$. At the same time, the Poisson's ratios for sintered AgNP and ECA have to be carefully assessed by taking into account the effect of porosity. Based on image analysis to statistically compare the areas of voids and solids in Fig. 1, the porosities are calculated as $5.66 \%$ and $22.9 \%$ for sintered AgNP and ECA, respectively. Hirose et al. [19] studied the relationship between Poisson's ratio and porosity of sintered steel powders and found that the Poisson's ratio was affected significantly by porosity but marginally influenced by the sintering atmosphere. As there is no such published experimental studies for sintered silver nanoparticles, the relationship between Poisson's ratio and porosity as found by Hirose et al. [19] is adopted for the similar porosity of sintered silver nanoparticles. In light of the Poisson's ratio of 0.37 for silver, a reduction of $10 \%$ is applied for ECA and the corresponding Poisson's ratio is 0.333 with porosity of about $22.9 \%$. As for sintered AgNP, a reduction of $1.6 \%$ is applied and the corresponding Poisson's ratio is 0.364 with porosity of about $5.66 \%$.

\section{Results}

After achieving the applied strain rate, the indenter was accurately controlled by Nano Indenter G200 to penetrate into the tested material. Thus, an initial desired strain rate can be applied and subsequently adjusted to another strain rate at a certain penetration depth. It can facilitate the investigation on nanomechanical behavior of interested material at the same location of the sample. Fig. 3(a) shows the applied strain rate history in the current study. Two strain rates of 0.1 $\mathrm{s}^{-1}$ and $0.2 \mathrm{~s}^{-1}$ were applied at independent indentations with three strain-rate jumps at the indentation depths of $1100 \mathrm{~nm}, 1400 \mathrm{~nm}$ and $1700 \mathrm{~nm}$, respectively. During each jump, the target strain rate lasts for $150 \mathrm{~nm}$ and the applied strain rate restores to the initial value for the 

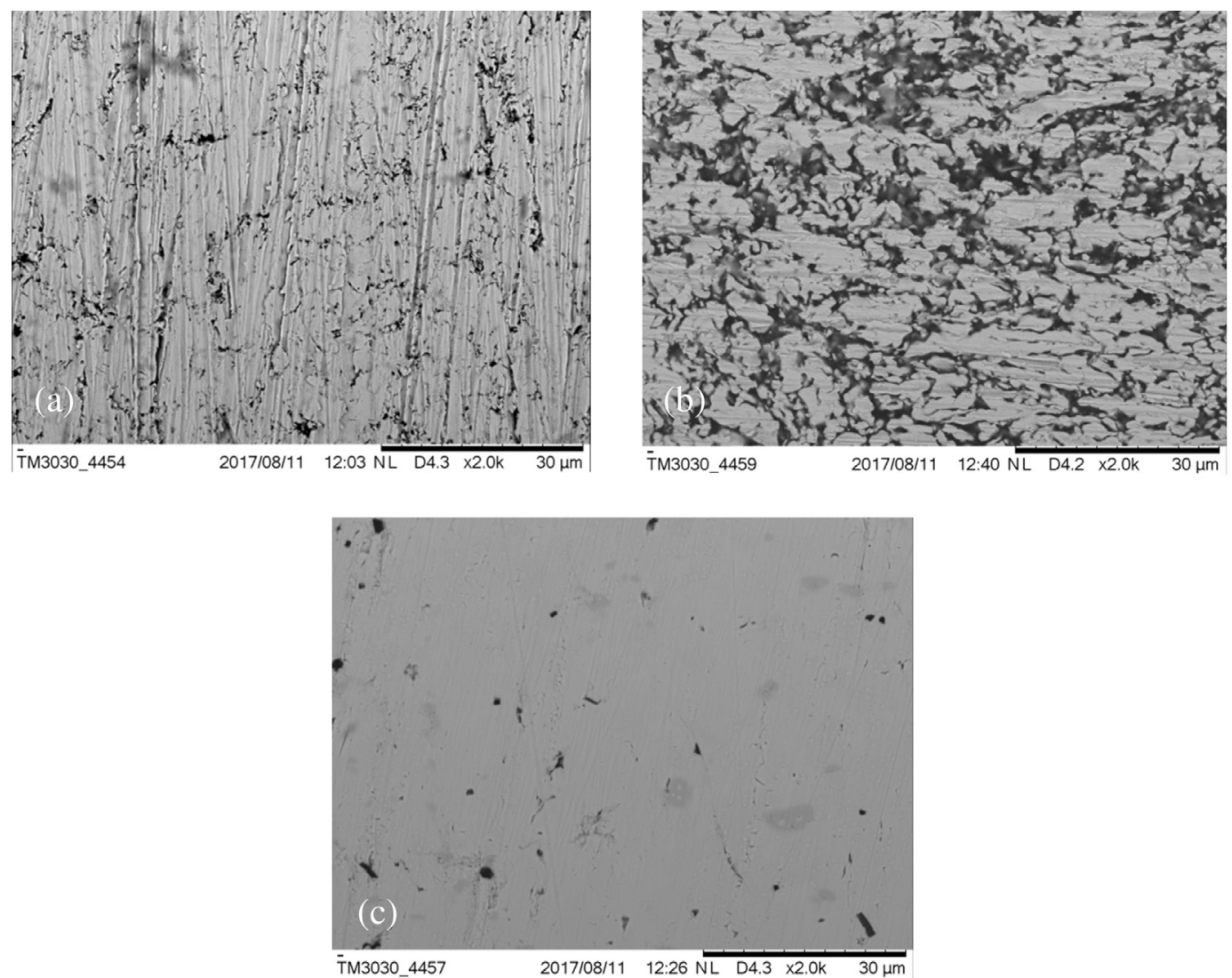

Fig. 1. SEM images at the magnification of 2000 times. (a) Sintered AgNP; (b) Sintered ECA; (c) SAC305 solder.
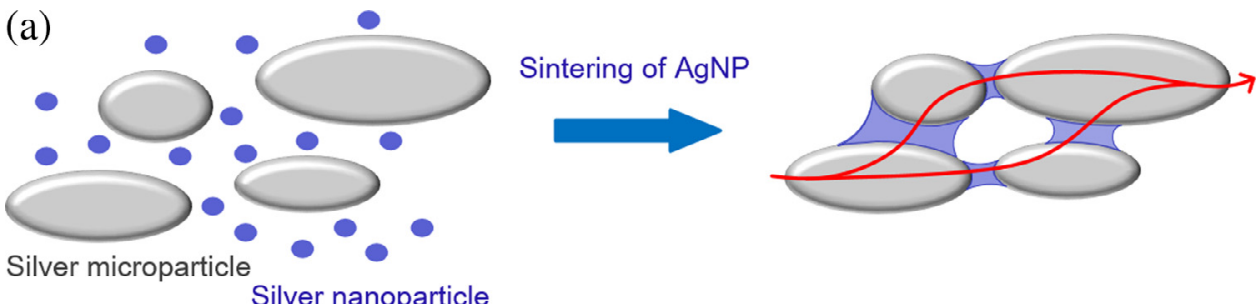

(b)
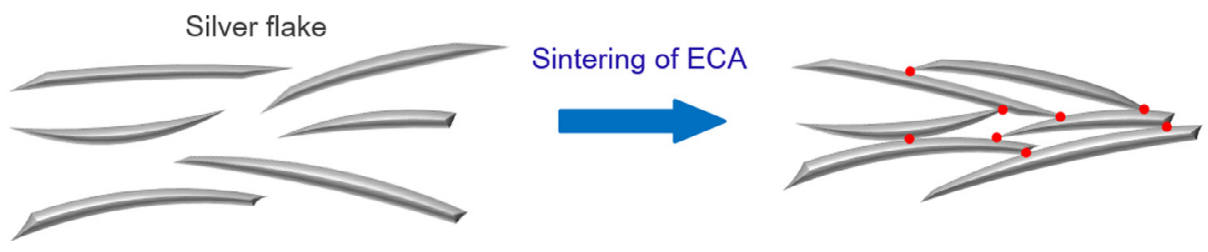

Fig. 2. Sintering mechanism. (a) AgNP; (b) ECA.

next indentation depth of $150 \mathrm{~nm}$. Fig. 3(b) shows the corresponding indentation load-penetration depth responses of the AgNP solder sample with the applied strain rate histories shown in Fig. 3(a). The maximum indentation depth was set to $2000 \mathrm{~nm}$. The indenter was held for $20 \mathrm{~s}$ at the maximum depth to alleviate the creep effect prior to the unloading stage. In terms of the local response in the indentation load-depth curve, a downward kink is observed results from the jump to a higher strain rate, while an upward kink appears caused by the jump to a lower strain rate. This unique property can be taken as a criterion to judge the positive strain rate sensitivity effect of indentation to the materials. The kinks in the indentation load-depth responses coincide with the jump of applied strain rate in Fig. 3(a). At the moment of indentation strain-rate jump, a load transition was found to exist with a self-similar behavior. This typical characteristics is in accordance to the finding by Lucas and Oliver [20].

The loading stages of sintered AgNP are compared in Fig. 4 with those of sintered ECA and SAC305 samples at the applied strain rate of $0.1 \mathrm{~s}^{-1}$ and $0.2 \mathrm{~s}^{-1}$. It is observed that the applied indentation load on the indenter for AgNP sample is greater, which means a higher hardness of sintered AgNP will be expected. Furthermore, the effect of strain rate is more significant for sintered AgNP, while sintered ECA is almost insensitive to the applied strain rate. This is speculated to be induced by the microstructural difference between sintered AgNP and ECA samples 

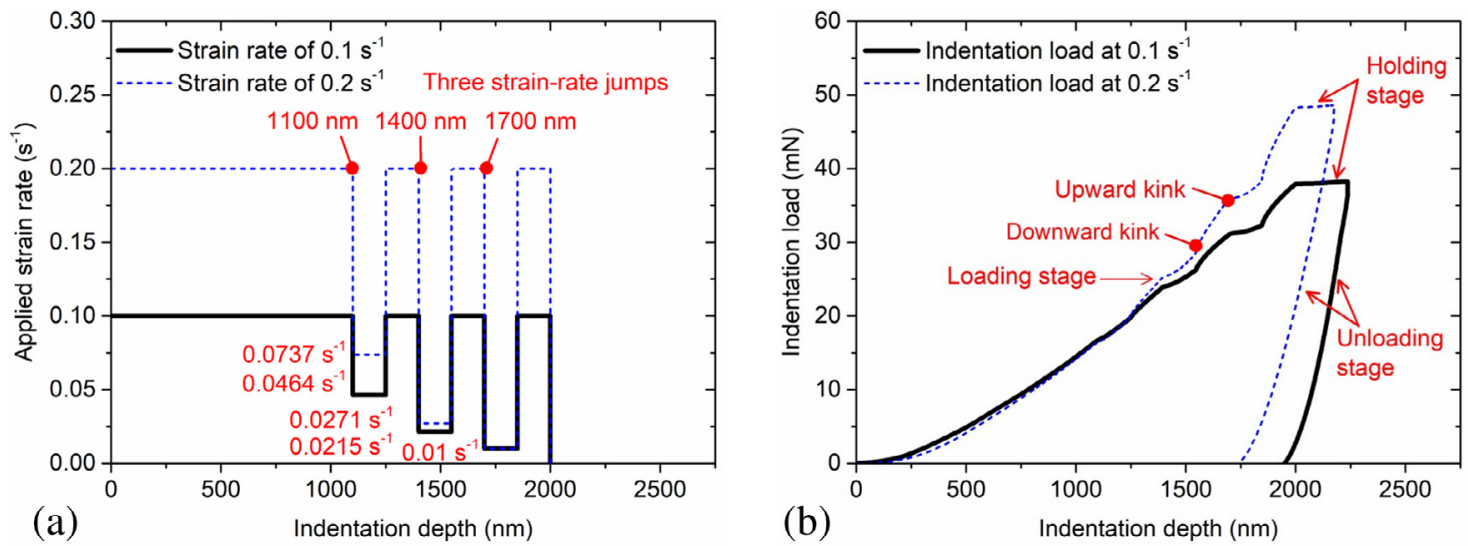

Fig. 3. Applied strain-rate jump nanoindentation on the AgNP solder sample. (a) Applied strain rate history; (b) Indentation load-depth response.

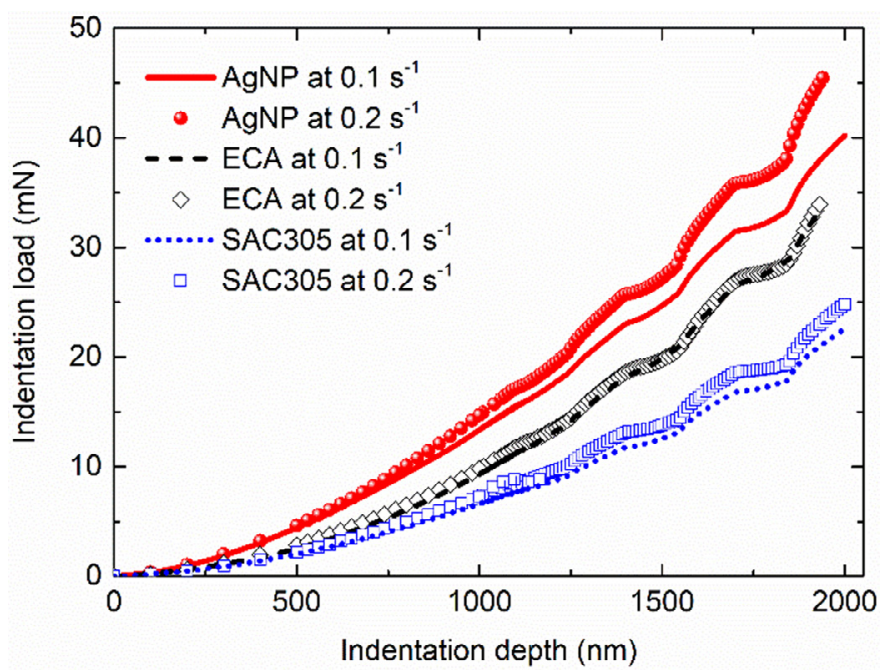

Fig. 4. Averaged indentation load-depth response of material samples at different applied strain rates during loading stage.

as shown in Fig. 1(a) and (b). Among the samples, the indentation load of SAC305 is the lowest, especially when a lower strain rate is applied.

In the current study, continuous stiffness measurement (CSM) technique $[21,22]$ is utilized to press a Berkovich indenter into contact of the sintered AgNP material sample, so that the contact force and other material properties (e.g., Young's modulus and hardness) can be measured as a continuous function of the indentation depth. Specifically, a small oscillation is superimposed to the indentation load. Meanwhile, the amplitude of force oscillation is controlled by a frequency-specific amplifier or a "lock-in amplifier" to ensure the amplitude of displacement oscillation remains a constant $5 \mathrm{~nm}$ at a driving frequency $f$ of $45 \mathrm{~Hz}$. Based on the harmonic oscillator, the contact stiffness $K_{c}$ was provided by Hay et al. [21] as:

$K_{c}=1 /\left[\frac{1}{\left(F_{0} / z_{0}\right) \cos \phi-\left.\left(F_{0} / z_{0}\right) \cos \phi\right|_{\text {free }}}-\frac{1}{K_{f}}\right]$

where $K_{f}$ is the elastic stiffness of the frame, $\phi$ is the phase angle by which the response lags the excitation, and $z_{0} / F_{0}$ is the dynamic compliance to represent the ratio of the displacement oscillation to the applied excitation. It should be noted that the subscript free indicates that the resonant (or natural) frequency of the indenter is in its free-hanging state, thus the term $\left.\left(F_{0} / z_{0}\right) \cos \phi\right|_{\text {free }}$ can be determined as $K-m \omega^{2}$, where $K$ is the stiffness of the spring supporting the indenter shaft, $m$ is the indenter mass, $\omega=2 \pi f$ represents the angular frequency of the indenter oscillates. The values of spring stiffness and indenter mass can be calibrated by the equipment manufacturer. Furthermore, the reduced Young's modulus $E_{r}$ can be determined by Eq. (2) [16]:

$E_{r}=\frac{\sqrt{\pi}}{2 \beta} \frac{K_{c}}{\sqrt{A_{c}}}$

where $A_{c}=24.56 h_{c}^{2}$ is the projected contact area at the contact depth $h_{c}$ and the shape constant $\beta=1.034$ for a Berkovich tip. In addition, the hardness can be conveniently calculated by dividing the indentation load by the square of indentation depth. The curves of hardness and Young's modulus as a function of indentation depth for the sintered AgNP are demonstrated in Fig. 5. This CSM technique can effectively avoid the scatter of reduced modulus measured at the initial gradient of the unloading curve of nanoindentation response. Fig. 6 provides the averaged results of Young's modulus and hardness for sintered AgNP, sintered ECA and SAC305, respectively, subjected to different indentation strain rates.

As ten indentations are repeated for each material and strain rate, the nanomechanical response can be accurately interpolated with a sufficient number of discrete data points for each indentation and the averaged values between $500 \mathrm{~nm}$ and $2000 \mathrm{~nm}$ are provided in Fig. 6. Accompanied with the applied strain-rate jump, the resulting variation of hardness and Young's modulus can be clearly demonstrated. It is found that the nanomechanical properties of all solder materials of interest change synchronously with strain rate.

Regarding the hardness in Fig. 6(a), sintered AgNP is more sensitive to the applied strain-rate jumps. The effect of strain rate for sintered ECA and SAC305 is negligible. By comparing the strain rate from the initial value of $0.2 \mathrm{~s}^{-1}$ to $0.1 \mathrm{~s}^{-1}$ at the indentation depth of $1700 \mathrm{~nm}$, the difference of hardness is about $0.075 \mathrm{GPa}$ for sintered AgNP, 0.007 GPa for sintered ECA, and $0.029 \mathrm{GPa}$ for SAC305. It should be noted that the effect of strain-rate jump for SAC305 solder seems to be moderate in terms of absolute value; however, the ratio between the hardness values before and after strain-rate jump is still high, especially for the shallow indentations. Upon the strain-rate jump to a lower value, the value of hardness is generally reduced within a short transient period. The measured value for SAC305 solder is stably around $0.25-0.28 \mathrm{GPa}$ for the strain rate of $0.1 \mathrm{~s}^{-1}$ and $0.2 \mathrm{~s}^{-1}$, which is in good accordance with the reported values of hardness [23]. The hardness is approaching 0.50-0.60 GPa for sintered AgNP and about 0.46 GPa for sintered ECA subjected to the strain rates of $0.1 \mathrm{~s}^{-1}$ and $0.2 \mathrm{~s}^{-1}$.

Compared with hardness, the effect of strain-rate jump on Young's modulus in Fig. 6(b) is moderate. In particular, the Young's modulus slightly decreases with the increase of indentation depth for sintered AgNP and ECA. However, the sintered AgNP is more sensitive to the applied strain rate compared with sintered ECA. This is ascribed to the densification of sintered AgNP during the indentation and correspond- 

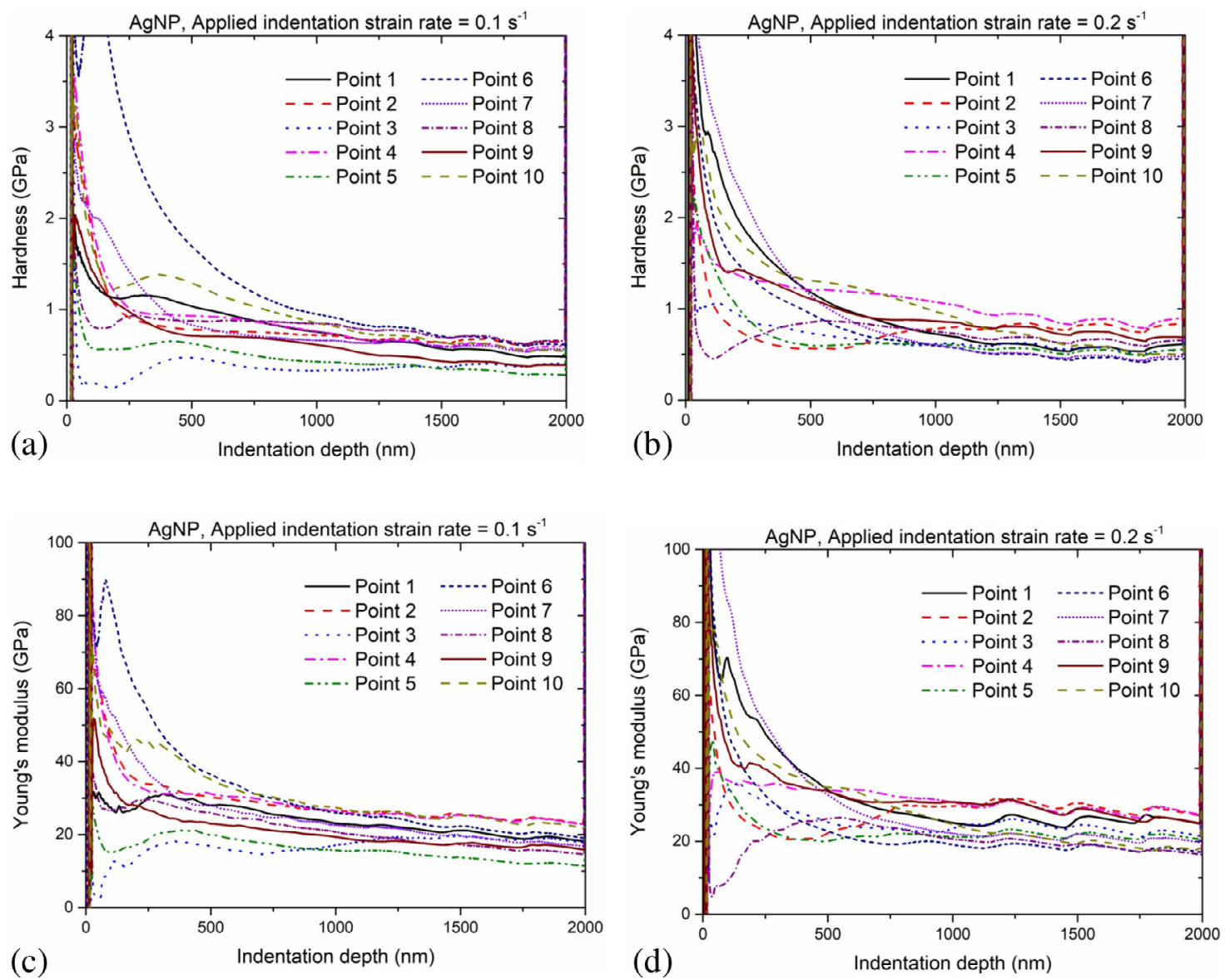

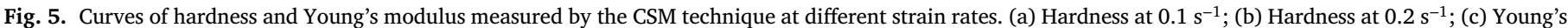
modulus at $0.1 \mathrm{~s}^{-1}$; (d) Young's modulus at $0.2 \mathrm{~s}^{-1}$.
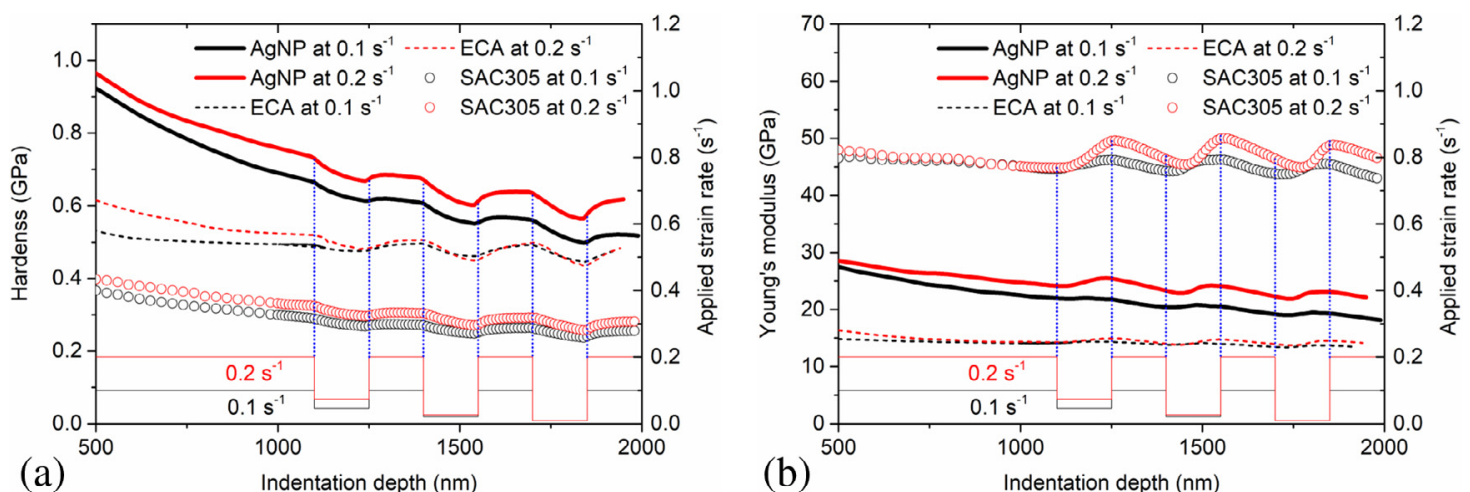

Fig. 6. Variation of hardness and Young's modulus of solder materials subjected to different applied strain rates. (a) Hardness; (b) Young's modulus.

ingly the local deformation will be more sensitive to the applied strain rate. Additionally, for SAC305 solder, when the indentation depth is greater than $1000 \mathrm{~nm}$, the measured Young's modulus is strongly dependent on the applied strain rate (i.e., $44.5 \mathrm{GPa}$ and $47.0 \mathrm{GPa}$ for the strain rates of $0.1 \mathrm{~s}^{-1}$ and $0.2 \mathrm{~s}^{-1}$ ) but insensitive to the indentation depth. These modulus values are at the same level of SAC305 solder with the Young's modulus determined by indentation tests [12], which validates the objectiveness of the present experimental approach for all samples.

\section{Discussion}

As deduced by Alkorta et al. [17], the applied strain rate $\dot{P} / P$ is closely related to the effective strain rate $\dot{\varepsilon}$ as shown in Eq. (3). The hardness is not significantly time-dependent and the term $\dot{H} / H$ can be assumed to be zero, so the applied strain rate $\dot{P} / P$ is approximately two times of the effective strain rate $\dot{\varepsilon}$.

$\frac{\dot{P}}{P}=\frac{\dot{H}}{H}+2 \frac{\dot{h}}{h} \approx 2 \frac{\dot{h}}{h}=2 \dot{\varepsilon}$

where $P$ is the applied indentation load, $H$ is the hardness, and $h$ is the indentation depth with the dot notation representing the rate of corresponding variables. It should be noted that the effective strain rate for a power-law creeping material obeys the relationship in Eq. (4) with 

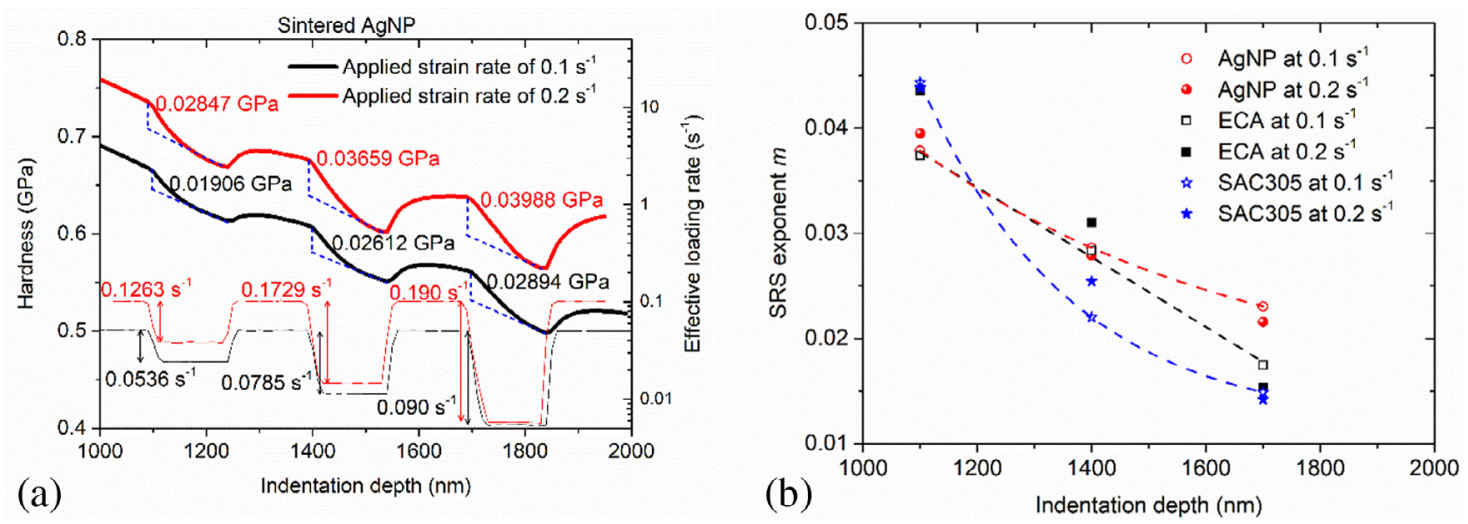

Fig. 7. Calculation of SRS exponent. (a) Variation of hardness and the corresponding strain rate; (b) SRS exponent.

hardness. With the definition of hardness $H=P / h^{2}$, the SRS exponent $m$ can be determined by Eq. (5).

$H=B \dot{\varepsilon}^{m}$

$m=\frac{d \ln (H)}{d \ln (\dot{\varepsilon})}$

Considering the strain-rate jump, the calculation of SRS exponent $m$ can be expressed in the form of Eq. (6), where the subscripts $i$ and $j$ represent the location before and after the rate-jump. This derivation has been elaborated by Alkorta et al. [17].

$m=\frac{\ln \left(H_{j}\right)-\ln \left(H_{i}\right)}{\ln \left(\dot{\varepsilon}_{j}\right)-\ln \left(\dot{\varepsilon}_{i}\right)}=\frac{\ln \left(H_{j} / H_{i}\right)}{\ln \left(\dot{\varepsilon}_{j} / \dot{\varepsilon}_{i}\right)}$

In order to calculate the SRS exponent $m$, the relationships between hardness and effective strain rate are plotted in Fig. 7(a) with the results, for example, of sintered AgNP. After similar calculations for sintered ECA and SAC305, the obtained SRS exponents are compared with different applied strain rates in Fig. 7(b). Additionally, the SRS exponents at the strain rate of $0.1 \mathrm{~s}^{-1}$ are numerically fitted. It is found that the SRS exponents of all solder materials in the present work decrease at a greater indentation depth. Compared with sintered ECA and SAC305, the SRS exponent $m$ of sintered AgNP decreases more mildly and approaches to a stable value with the increase of penetration depth at the same indentation strain rate, which means that the variation rate of hardness within sintered AgNP is effectively stabilized along the indenting direction according to Eq. (3). This behavior is similar to that for SAC305 solder material. The underlying reason is that when the indentation is sufficiently large, the deformation field under the indenter becomes self-similar and the hardness is therefore independent of indentation depth.

In contrast, the SRS exponent of sintered ECA is almost linearly reduced when the indentation depth increases. This phenomenon can be further explained by taking into account the sensitivity of hardness to porosity. Through finite element analyses based on the Gurson model for plastic deformation of ductile porous materials, Chen et al. [24] found that the porosity reduces the hardness of porous materials due to densification. As shown in Fig. 7(b), the SRS exponent of sintered ECA is much smaller than that of sintered AgNP at the indentation depth of $1700 \mathrm{~nm}$. According to Eq. (3), a smaller exponent value results from the smaller variation of hardness at the same indentation depth and strain jump. Therefore, compared with sintered AgNP, higher porosity in sintered ECA leads to greater densification and smaller hardness variation.

The creep behavior can be described by a power law equation in the form of Eq. (7) at an isothermal condition,

$\dot{\varepsilon}_{c}=A \sigma^{n}$

$\sigma \propto P / h^{2}$ where $A$ is a material constant, $\sigma$ is the applied stress which can be related with indentation depth $h$ in Eq. (8) and $n$ is the stress exponent which generally indicates the deformation mechanism of creep behavior. Therefore, the stress exponent can be numerically fitted according to Eq. (7) in the log-log diagram between creep strain rate and applied stress.

As shown in Fig. 3, a holding stage of $20 \mathrm{~s}$ is applied in the indentation load-depth history to alleviate the creep effect prior to the unloading stage upon the maximum depth of $2000 \mathrm{~nm}$. As the applied load on indenter is kept the same during the holding stage, creep deformation is induced as displayed in Fig. 8(a) and the corresponding creep strain rate $\dot{\varepsilon}_{c}$ is obtained in Fig. 8(b) based on Eq. (9) as defined by Mayo and Nix [25].

$\dot{\varepsilon}_{c}=\frac{\dot{h}}{h}=\frac{d h / d t}{h}$

where $h$ represents the accumulative indentation depth and $t$ is the holding time.

As shown in Fig. 8(a), the creep displacement of SAC305 solder sample is much greater and the effect of strain rate is more significant. This phenomenon agrees well with the indentation findings by Xiao et al. [23] for SAC305 solder. On the other hand, the creep displacement of sintered AgNP and ECA remains at a similar level despite of the applied strain rate. Based on the creep displacement-holding time relationships in Fig. 8(b), the creep strain rate decays exponentially with the increase of holding time. Nevertheless, the strain rate effect on the creep strain rate is more pronounced at the beginning of holding stage. That is, the difference due to the applied strain rate is $0.011 \mathrm{~s}^{-1}$ for AgNP, $0.021 \mathrm{~s}^{-1}$ for ECA and $0.028 \mathrm{~s}^{-1}$ for SAC305, respectively. Subsequently, the difference of creep strain rate is negligible after a sufficient holding time, which can be found in the inset of Fig. 8(b). Although there are slight fluctuations, the creep strain rate is stabilized at $0.0011 \mathrm{~s}^{-1}$ for both AgNP and ECA, and $0.0026 \mathrm{~s}^{-1}$ for SAC305, respectively. It should be noted that compared with the holding stage of $10 \mathrm{~s}$, which has been used to investigate the creep behavior of SAC305 solder [26], the creep rate with a longer holding duration as applied in the current study is further reduced as expected.

Numerical regressions were performed by using the linear function in OriginPro 9.0 at the beginning and ending portions of holding stage in the log-log relationships between creep strain rate and applied stress. As displayed in Fig. 9(c), the obtained stress exponents for SAC305 solder under the applied strain rates of $0.1 \mathrm{~s}^{-1}$ and $0.2 \mathrm{~s}^{-1}$ are 16.2 and 19.0 at the beginning of holding stage, 12.7 and 13.6 at the end of the holding stage. The stabilized values of stress exponents after sufficient accumulation of creep deformation are indicative of dislocation climb as the deformation mechanism, which are in good accordance to other creep behavior investigations of lead-free solders based on the tensile and strain-rate-change tests [27]. The experimental and analytical approaches in the present study are highly reliable to evaluate the nanome- 

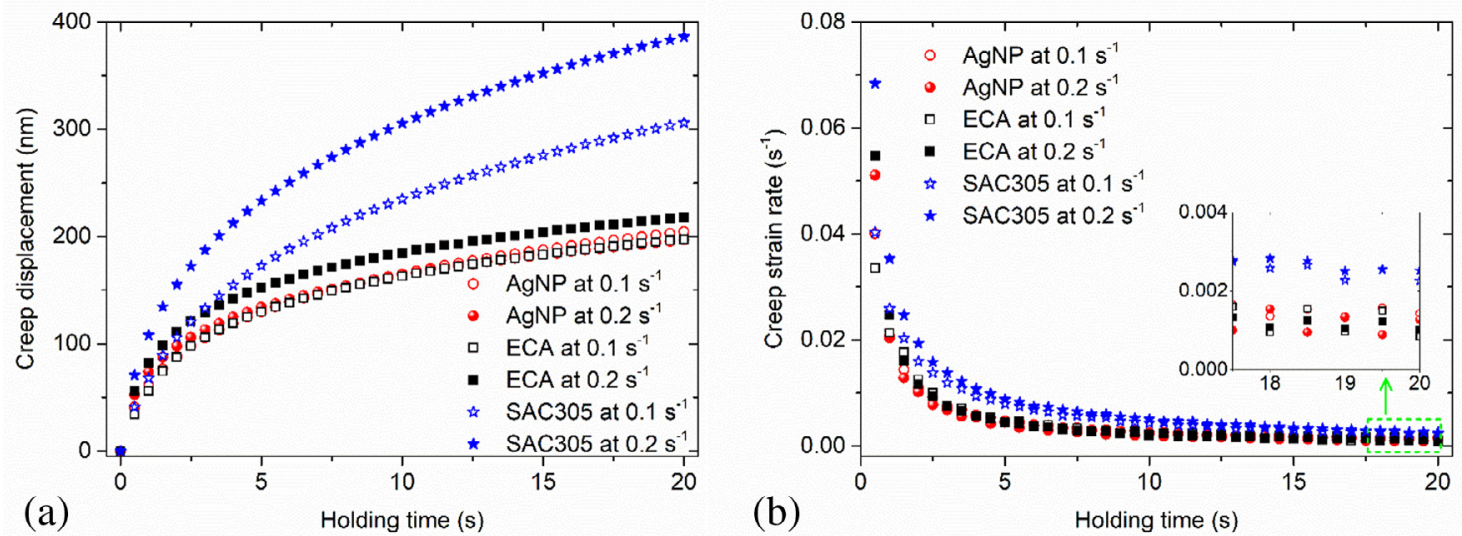

Fig. 8. Creep behavior during holding stage. (a) Creep displacement; (b) Creep strain rate.
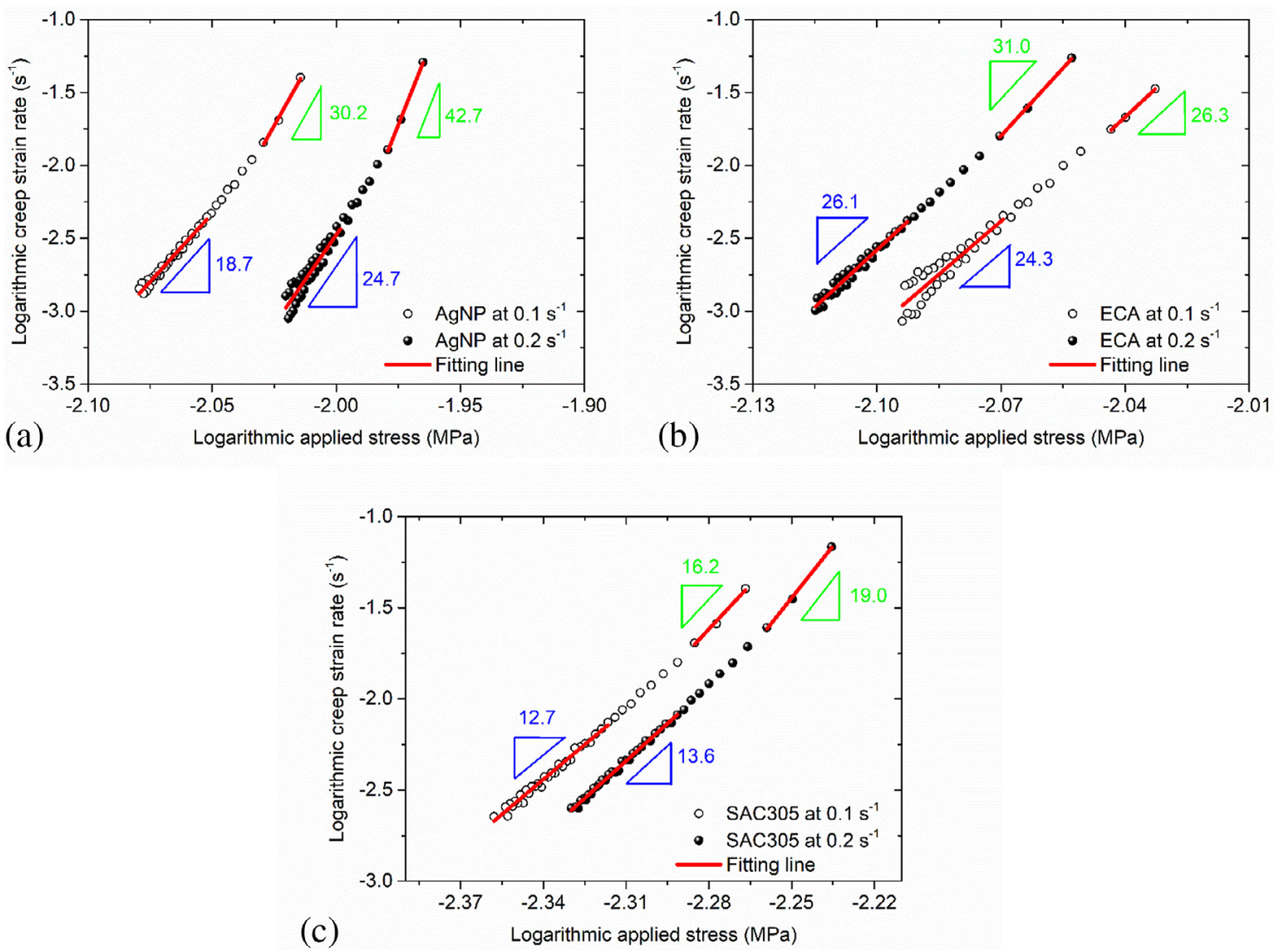

Fig. 9. Stress exponent of solder materials subjected to different applied strain rates. (a) Sintered AgNP; (b) Sintered ECA; (c) SAC305.

chanical properties of solder materials. Nevertheless, Phani and Oliver [28] found that using a Berkovich indenter, the indentation that induces a nominal strain of $8 \%$ and the indentation creep behavior can only be compatible with the uniaxial creep tests at a similar strain. Therefore, the creep properties of solder materials measured by indentation approaches should be further investigated to address the compatibility with the creep behavior of macroscopic applications at different strain ranges.

Similarly, the variation of stress exponents for sintered AgNP and ECA are provided in Fig. 9(b) and (c) for both the beginning and ending portions of the $20 \mathrm{~s}$ holding stage. Furthermore, the stabilized stress exponents are 18.7 and 24.7 for sintered AgNP and 24.3 and 26.1 for sintered ECA under the applied strain rates of $0.1 \mathrm{~s}^{-1}$ and $0.2 \mathrm{~s}^{-1}$, respectively. The stress exponents of sintered AgNP and ECA are greater than
SAC305 solder, which means that the creep strain rates of sintered silver materials are more sensitive to the applied stress. More importantly, due to the definition of SRS exponent $m$ in Eq. (2) and stress exponent $n$ in Eq. (5), a reciprocal relationship seems to be obeyed between $m$ and $n$. However, the reciprocals of stress exponents at the beginning of the holding stage shown in Fig. 9 are at the same level of those values in Fig. 7(b), however, there will be an obviously increasing trend until the ending of the holding stage. During the holding stage, the accumulated viscous deformation is transformed into larger creep deformation [23], thus the creep deformation is approaching a stabilized state to be more sensitive to the applied stress. In addition, the SRS exponent $m$ is measured in the loading stage, and the stress exponent $n$ is numerically fitted in the holding stage. It is hypothesized that the deformation mechanisms of sintered AgNP with a complicated porous microstruc- 
ture are different at the loading and holding stages, therefore further investigations should be performed. Alternatively, if much longer holding durations can be achieved to obtain a sufficient steady-state creep deformation and more importantly, the creep behavior of sintered AgNP can be satisfactorily described by a power-law relationship between the flow stress and creep strain rate, a mathematical approach proposed by Chinh and Szommer [29] can be conveniently adopted to evaluate the SRS exponent at the holding stage by numerical regressions.

\section{Conclusions}

The strain rate sensitivity of sintered AgNP is investigated by nanoindentation experiments with strain-rate jumps adopting the CSM technique. With the increasing indentation depth of sintered AgNP greater than $1000 \mathrm{~nm}$, the hardness approaches $0.50-0.60 \mathrm{GPa}$ and the Young's modulus is $18.1 \mathrm{GPa}$ and $22.0 \mathrm{GPa}$ at the strain rates of $0.1 \mathrm{~s}^{-1}$ and 0.2 $\mathrm{s}^{-1}$, respectively. Compared with sintered ECA and SAC305 solder, the hardness of sintered AgNP is greater and strongly sensitive to the applied strain rate, while the strain rate effect on Young's modulus of sintered AgNP is moderate. SRS exponents generally decrease with larger indentation depths. Stable values are achieved for sintered AgNP and SAC305 solder because the variation rate of hardness is stabilized at the indentation depths between $1000 \mathrm{~nm}$ and $2000 \mathrm{~nm}$. The observed linear decreasing of SRS exponent for sintered ECA is attributed to the reduced variation of hardness with the densification of higher porosity. Compared with the SAC305 solder, the creep displacements of sintered AgNP and ECA are relatively insensitive to the applied strain rates and the creep strain rates are much lower especially at the beginning of the holding stage. The stress exponents of AgNP are determined to be 18.7 and 24.7 at the strain rates of $0.1 \mathrm{~s}^{-1}$ and $0.2 \mathrm{~s}^{-1}$, respectively.

\section{Acknowledgments}

This work was supported by the National Natural Science Foundation of China (Nos. 51508464 and 11772257), the Natural Science Foundation of Shaanxi Province (No. 2017JM1013), the Fundamental Research Funds for the Central Universities (No. 3102016ZY017), and the Astronautics Supporting Technology Foundation of China(No. 2017-HT-XG).

\section{References}

[1] European Union 95/EC of the European Parliament and of the Council of 27 January 2003 on the restriction of the use of certain hazardous substances in electrical and electronic equipment. Off J Eur Union 2003:19-23 L37.

[2] Yao Y, Long X, Keer LM. A review of recent research on the mechanical behavior of lead-free solders. Appl Mech Rev 2017;69:040802.

[3] Maruyama M, Matsubayashi R, Iwakuro H, Isoda S, Komatsu T. Silver nanosintering: a lead-free alternative to soldering. Appl Phys A 2008;93:467-70.

[4] Zuruzi AS, Siow KS. Electrical conductivity of porous silver made from sintered nanoparticles. Electron Mater Lett 2015;11:308-14.
[5] Yu H, Li L, Zhang Y. Silver nanoparticle-based thermal interface materials with ultra-low thermal resistance for power electronics applications. Scr Mater 2012;66:931-4.

[6] Wang S, Ji H, Li M, Wang C. Fabrication of interconnects using pressureless low temperature sintered Ag nanoparticles. Mater Lett 2012;85:61-3.

[7] Chua ST, Siow KS. Microstructural studies and bonding strength of pressureless sintered nano-silver joints on silver, direct bond copper (DBC) and copper substrates aged at $300{ }^{\circ} \mathrm{C}$. J Alloy Compd 2016;687:486-98.

[8] Paknejad SA, Mansourian A, Greenberg J, Khtatba K, Parijs LV, Mannan SH. Microstructural evolution of sintered silver at elevated temperatures. Microelectron Reliab 2016;63:125-33.

[9] Yang CX, Li X, Lu GQ, Mei YH. Enhanced pressureless bonding by Tin Doped Silver Paste at low sintering temperature. Mater Sci Eng, A 2016;660:71-6.

[10] Gadaud P, Caccuri V, Bertheau D, Carr J, Milhet X. Ageing sintered silver: relationship between tensile behavior, mechanical properties and the nanoporous structure evolution. Mater Sci Eng, A 2016;669:379-86.

[11] Leslie D, Dasgupta A, Morillo C. Viscoplastic properties of pressure-less sintered silver materials using indentation. Microelectron Reliab 2017;74:121-30.

[12] Long X, Wang S, Feng Y, Yao Y, Keer LM. Annealing effect on residual stress of Sn-3.0Ag-0.5Cu solder measured by nanoindentation and constitutive experiments. Mater Sci Eng, A 2017;696:90-5.

[13] Long X, Tang W, Xia W, Wu Y, Ren L, Yao Y. Nanoindentation response of pressureless sintered silver nanoparticles. 19th Electron Packaging Technol Conf; 2017.

[14] Cheng S, Huang CM, Pecht M. A review of lead-free solders for electronics applications. Microelectron Reliab 2017;75:77-95.

[15] Maier V, Durst K, Mueller J, Backes B, Höppel HW, Göken M. Nanoindentation strain-rate jump tests for determining the local strain-rate sensitivity in nanocrystalline Ni and ultrafine-grained Al. J Mater Res 2011;26:1421-30.

[16] Oliver WC, Pharr GM. Improved technique for determining hardness and elastic modulus using load and displacement sensing indentation experiments. J Mater Res 1992;7:1564-83.

[17] Alkorta J, Martínez-Esnaola JM, Gil Sevillano J. Critical examination of strain-rate sensitivity measurement by nanoindentation methods: application to severely deformed niobium. Acta Mater 2008;56:884-93.

[18] Nguyen TT, Yu D, Park SB. Characterizing the Mechanical Properties of Actual SAC105, SAC305, and SAC405 Solder Joints by Digital Image Correlation. J Electron Mater 2011;40:1409-15.

[19] Hirose N, Asami J, Fujiki A, Oouchi K. Poisson's ratio of sintered materials for structural machine parts. Powder Metall Technol 2004;51:515-21.

[20] Lucas BN, Oliver WC. Indentation power-law creep of high-purity indium. Metall Mater Trans A 1999;30:601-10.

[21] Hay J, Agee P, Herbert E. Continuous stiffness measurement during instrumented indentation testing. Exp Tech 2010;34:86-94.

[22] Chang C, Garrido MA, Ruiz-Hervias J, Rodríguez J. On the possibility of reducing the pile-up effect on the Berkovich instrumented indentation tests. Int J Mech Sci 2017;121:181-6.

[23] Xiao G, Yuan G, Jia C, Yang X, Li Z, Shu X. Strain rate sensitivity of Sn-3.0Ag-0.5Cu solder investigated by nanoindentation. Mater Sci Eng, A 2014;613:336-9.

[24] Chen X, Xiang Y, Vlassak JJ. Novel technique for measuring the mechanical properties of porous materials by nanoindentation. J Mater Res 2006;21:715-24.

[25] Mayo MJ, Nix WD. A micro-indentation study of superplasticity in Pb, Sn, and Sn-38 wt $\% \mathrm{~Pb}$. Acta Metall 1988;36:2183-92.

[26] Long X, Feng Y, Yao Y. Cooling and annealing effect on indentation response of lead-free solder. Int J Appl Mech 2017;9:1750057.

[27] Shohji I, Yoshida T, Takahashi T, Hioki S. Tensile properties of Sn-Ag based lead-free solders and strain rate sensitivity. Mater Sci Eng, A 2004;366:50-5.

[28] Phani PS, Oliver WC. A direct comparison of high temperature nanoindentation creep and uniaxial creep measurements for commercial purity aluminum. Acta Mater 2016;111:31-8

[29] Chinh NQ, Szommer P. Mathematical description of indentation creep and its application for the determination of strain rate sensitivity. Mater Sci Eng, A 2014;611:333-6 